This item was submitted to Loughborough's Research Repository by the author.

Items in Figshare are protected by copyright, with all rights reserved, unless otherwise indicated.

\title{
2D finite element analysis of thermally bonded nonwoven materials: continuous and discontinuous models
}

PLEASE CITE THE PUBLISHED VERSION

https://doi.org/10.1016/j.commatsci.2009.07.007

\section{PUBLISHER}

(C) Elsevier

\section{VERSION}

AM (Accepted Manuscript)

\section{PUBLISHER STATEMENT}

This work is made available according to the conditions of the Creative Commons Attribution-NonCommercialNoDerivatives 4.0 International (CC BY-NC-ND 4.0) licence. Full details of this licence are available at: https://creativecommons.org/licenses/by-nc-nd/4.0/

\section{LICENCE}

CC BY-NC-ND 4.0

\section{REPOSITORY RECORD}

Hou, Xiaonan, Memis Acar, and Vadim V. Silberschmidt. 2019. "2D Finite Element Analysis of Thermally Bonded Nonwoven Materials: Continuous and Discontinuous Models”. figshare. https://hdl.handle.net/2134/25651. 


\title{
2D finite element analysis of thermally bonded nonwoven materials: Continuous and discontinuous models
}

\author{
Xiaonan Hou *, Memiş Acar, Vadim V. Silberschmidt \\ Wolfson School of Mechanical and Manufacturing Engineering, Loughborough University, Leicestershire, LE11 3TU, UK
}

A B S T R A C T

\begin{abstract}
Due to a random structure of nonwoven materials, their non-uniform local material properties and nonlinear properties of single fibres, it is difficult to develop a numerical model that adequately accounts for these features and properly describes their performance. Two different finite element (FE) models - continuous and discontinuous - are developed here to describe the tensile behaviour of nonwoven materials. A macro-level continuum finite element model is developed based on the classic composite theory by treating the fibrous network as orthotropic material. This model is used to analyse the effect of thermally bonding points on the deformational behaviour and deformation mechanisms of thermally bonded nonwoven materials at macro-scale. To describe the effects of discontinuous microstructure of the fabric and implement the properties of polypropylene fibres, a micro-level discontinuous finite element model is developed. Applicability of both models to describe various deformational features observed in experiments with a real thermally bonded nonwoven is discussed.
\end{abstract}

\author{
Keywords: \\ Nonwovens \\ Finite element analysis \\ Random microstructure \\ Anisotropic behaviour
}

\section{Introduction}

Nonwovens are one of the fastest growing textile sectors with diverse applications. These applications can form an extensive list, ranging from baby diapers to high-performance geotextiles [1]. Therefore, one of the main requirements of the industry is good understanding of mechanical properties of such materials and the mechanism of their deformation at both microscopic and macroscopic levels. In last 40 years, many efforts have been made by researchers to understand the material's performance of nonwovens. One of the research challenges on the way to this understanding is to link the properties of fibres and the fabric's microstructure to the main mechanisms of material's deformation and failure.

Thermally-bonded nonwoven materials are manufactured by interlocking polymeric fibres using heat energy that partially melt the fibres in contact points during calendaring [2]. Some theoretical approaches to deformation of fibrous networks in 2D and 3D were developed (see, e.g. $[3,4]$ ). The mechanical properties of nonwoven materials are determined by their microstructure, fibre properties and bonding features. From Backer and Petterson's pioneering work [5], researchers have used considerable efforts

\footnotetext{
* Corresponding author. Tel.: +44 01509227566

E-mail address: x.hou@lboro.ac.uk (X. Hou).
}

to develop methods to describe the unique material. Basically, there are two most popular theories at present, and most of the researches are based on them. One is the orthotropic theory, which is used to predict directly the properties of a thermally bonded nonwoven at the macroscopic level [6]. Some researchers also use a classic orthotropic theory to model spun-bonded nonwovens [7]. The theory treats nonwoven materials as a composited system using the laminate composite theory and assumes for every layer of fibres a unique orientation.

Another theory is the web-cell one, which can introduce properties of fibres and the effect of microstructure into the analysis of nonwoven fabrics. This theory is based on the incremental deformation principle and predicts the material behaviour with the consideration of fibre properties, their orientation distribution function (ODF) and bonding deformation [8-10,11-16].

An adequate account for complex microstructures and properties of nonwovens is possible only within the framework of numerical simulations. But the use of traditional techniques, e.g. finite element analysis (FEA), is linked to serious challenges, the most important being discontinuity of nonwovens in contrast to standard structural materials. The existing literature on mechanics of fibrous networks is limited mainly to paper $[17,18]$.

Hence, only a few works dealing with the use of finite elements have been implemented to describe nonwoven materials. Mueller and Kochmann [19] used a FEA approach to simulate the tensile 
behaviour of thermally bonded nonwovens by modelling the single fibre as a spar, and the bond points were modelled using solid elements. Their work successfully involved a nonlinear behaviour and described plastic deformation of the material. But one of the disadvantages of their approach is that the model cannot incorporate information on the ODF since a real random microstructure of the fibrous network is presented with a periodic structure. To predict the tensile behaviour of adhesive point-bonded spunbond fabric, Limem and Warner [20] developed a truss-based model. They used one group of bond points and seven fibre groups to represent the structure of the fabric, and bond points were assumed to be interchangeable. Like the previous model, this model cannot describe some nonlinear phenomena of nonwovens due to its periodic structure. In 2006, the concept of the representative volume element (RVE) was used by Engelmayr and Sacks in their model of the flexural mechanics of nonwoven tissues [21].

The present paper suggests two FE models - a continuous model and a discontinuous one - to describe the material's tensile behaviour at both macro-scale and micro-scale.

\section{Thermally bonded nonwoven material: Microstructure and properties}

The thermally bonded nonwoven material (polypropylene fibres; density $20 \mathrm{gsm}$ ) is used in this study; its microstructure is shown in Fig. 1. The density unite of fabric is gsm, which is defined as gram per square meters. Therefore, the density of the nonwoven fabric in this research is extremely low. Void areas exist in the fibrous network making the material is discontinuous. It is clear that the nonwoven material includes two components: a fibrous web and bonding points. Rectangles, forming a regular pattern in Fig. 1a, are bonding points and other parts of nonwoven forms the fibrous web. Fig. 1b shows a bonding point, which is formed by partially melted and compressed fibres. As a result of this thermal process the areas of bonding points are much stiffer than the areas occupied by the fibrous web since the fibres in these points are glued together and their relative movements that are responsible for high stretching ability of the fibrous network are severely restricted.

As a result of the manufacturing process thermally bonded nonwovens are spatially non-uniform materials, as Fig. 2 vividly demonstrates. Another important manufacturing-induced property of the material is its anisotropy. In the manufacturing process, the fibres are assembled in the web plane randomly. Hence, in different directions of the plane of the fabric, different material properties are obtained. But due to the manufacturing process, the so called machine direction (MD) coinciding with the direction of formation of the band is a preferred direction of the material, and more fibres are assembled along this direction. The cross direction (CD) is perpendicular to MD; the smallest fraction of the fibres is assembled along it. Therefore, material properties in machine direction are much higher than those in cross direction (CD). Fig. 3 shows different stress-strain relationships for machine direction and cross direction for the studied nonwoven, and they are also used as input into continuous FE models. The traditional stress unit used for fabrics is Newton per millimeter of its length, which assumes the thickness of the material is constant. The details of our experimental studies of the thermally bonded nonwoven are given in [22].

\section{Continuous finite element model}

To describe the effect of bonding points and the anisotropic material properties of the nonwoven material, a continuous model is developed implementing the material features at macro-scale.

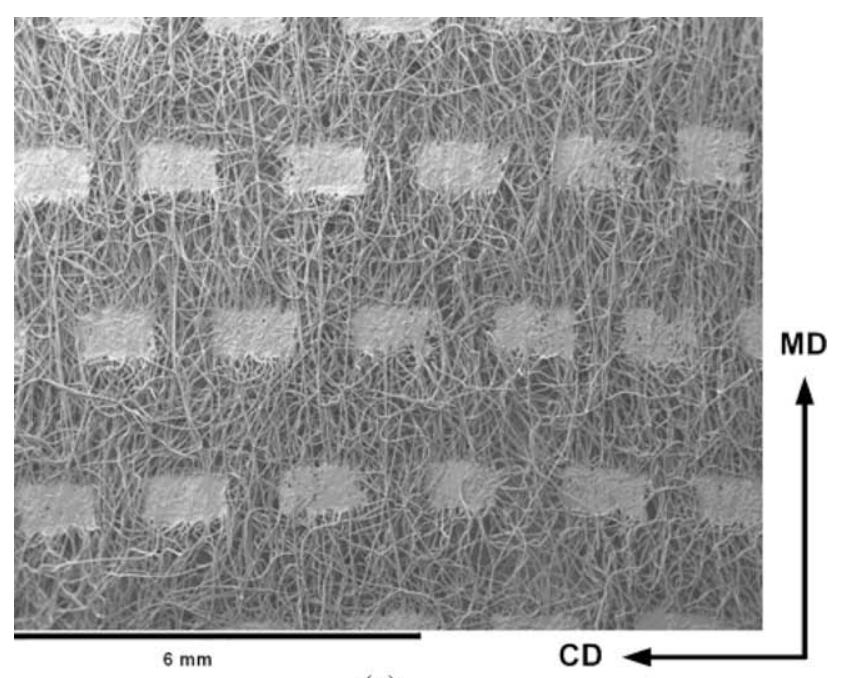

(a)

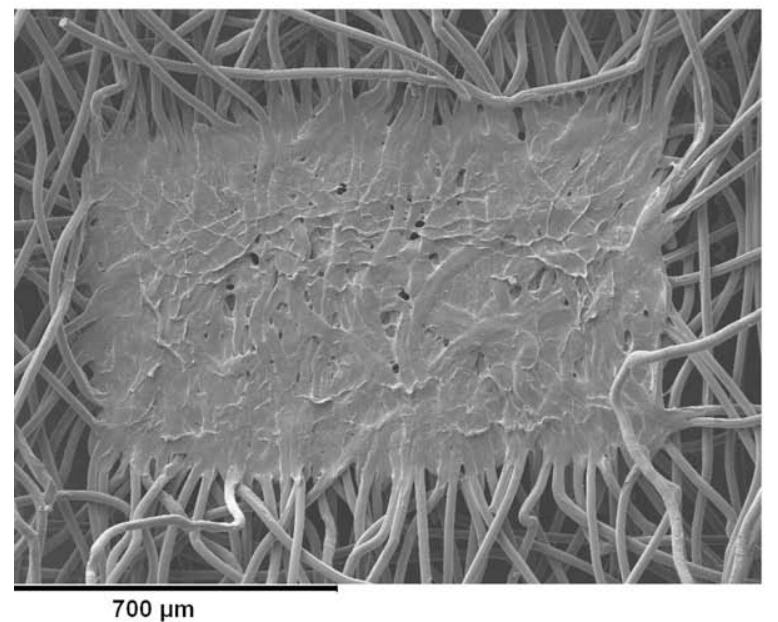

(b)

Fig. 1. SEM image of thermally bonded nonwoven material (20 gsm) (a) and bonding point (b).

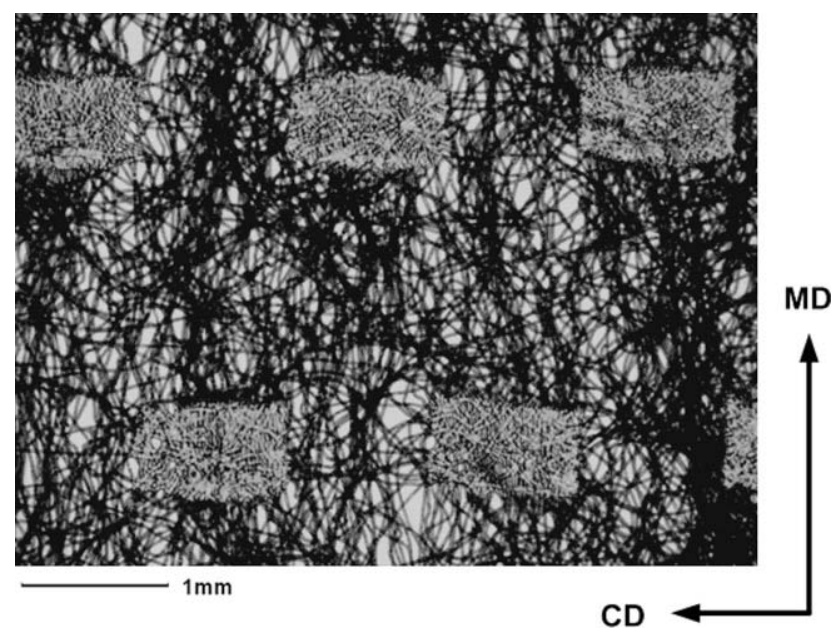

Fig. 2. Microscopic image of thermally bonded nonwoven with background light.

Different elasto-plastic material properties were incorporated in it for fibrous web and bonding points. Since it is rather cumbersome to determine the stiffness of bonding points, at this stage 


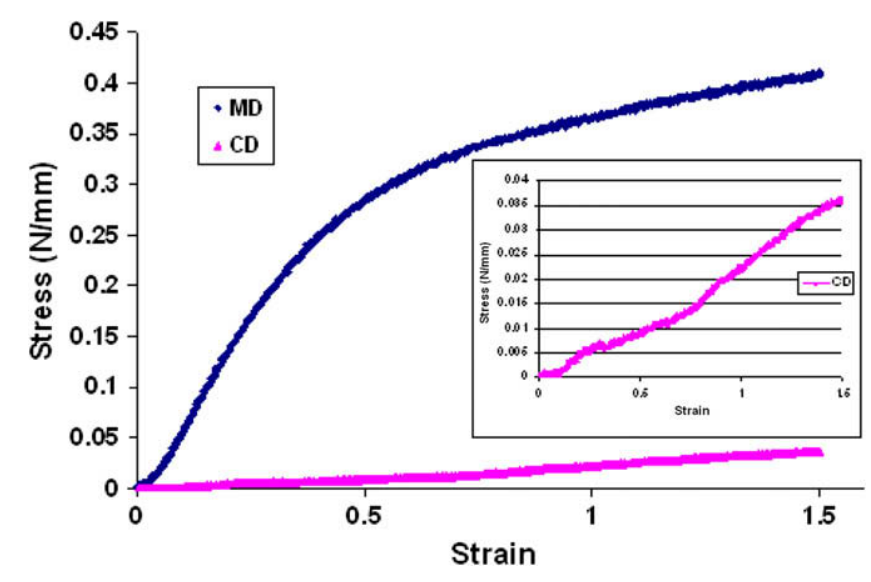

Fig. 3. Typical stress-strain relationships for thermally bonded nonwoven material in machine direction and cross direction.

of research it was assumed to be three times higher than that of the fibrous area. According to our experiment results of the nonwoven (Fig. 3). Material properties of the fibrous web and bonding points were calculated according to there area fraction in both machine direction and cross direction. Due to the character of the fabric, the material parameters for a through-thickness direction are difficult to measure. Therefore, in the model, they were assumed to be equal to those for the cross direction. The Poisson's ratio used in this model had a provisional value of 0.3 .

The geometry of the continuous model is shown in Fig. 4. The model is a two-dimension model, defined in $x-y$ plane. At initial moment $t=0$, the dimensions of the undeformed fabric are $25 \mathrm{~mm} \times 20 \mathrm{~mm}$. The dimensions of each bonding point are (width) $0.75 \mathrm{~mm} \times$ (length) $1.1 \mathrm{~mm}$. The spacing arrangement of bonding points is shown in Fig. 5 and the respective magnitudes are given in Table 1. Since bonding points in the model are not arranged symmetrically (Fig. 5), the meshing based on rectangular element causes their distortion around bonding areas in the model. Hence, triangular elements are chosen for the model. The real fabric is a very thin material, and thickness of the bonding points is even smaller than that of the fibrous areas. Therefore, ABAQUS quadratic triangle element STRI65 was used to simulate the fibrous web, and quadratic quadrilateral element S8R5 was used to model the bonding points [23]. Thickness of those two types of elements is assumed not to change during the analysis, matching our assumption for the experiments - usually, the fabric's thickness is not considered in the researches of nonwovens. But these elements still have their limitations in representing this low-density nonwoven materials; for instance, they are not appropriate to simulate the material's behaviour at high strain levels. So, at the next stage of our research, more advanced finite elements and the remeshing technique will be employed.

To simulate the tensile test, a set of boundary conditions was applied to the FE continuous model. The left boundary of the specimen (Fig. 4) was set as "ENCASTRE". The setting constrains all the degrees of freedom of the nodes in this boundary to simulate fixture of specimen in the jaws of the tester. A uniform displacement $(15 \mathrm{~mm})$ in $x$ direction was applied to the right boundary of the specimen to simulate its extension by the moving jaw.

The material properties of the model were defined according to the classic composite theory with orthotropic mechanical parameters taken from our experimental study. The results of simulations for both MD and CD models are shown in Fig. 6 for the axial strain. Different stress distributions are the apparent results of simulations, which are due to the different arrangements of bonding points along two principle directions in the nonwoven. In machine

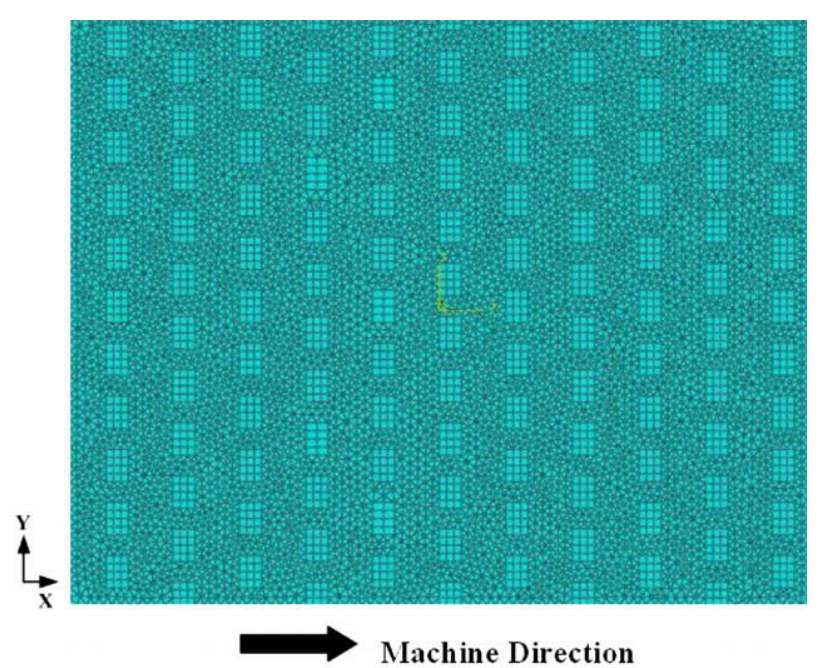

(a)

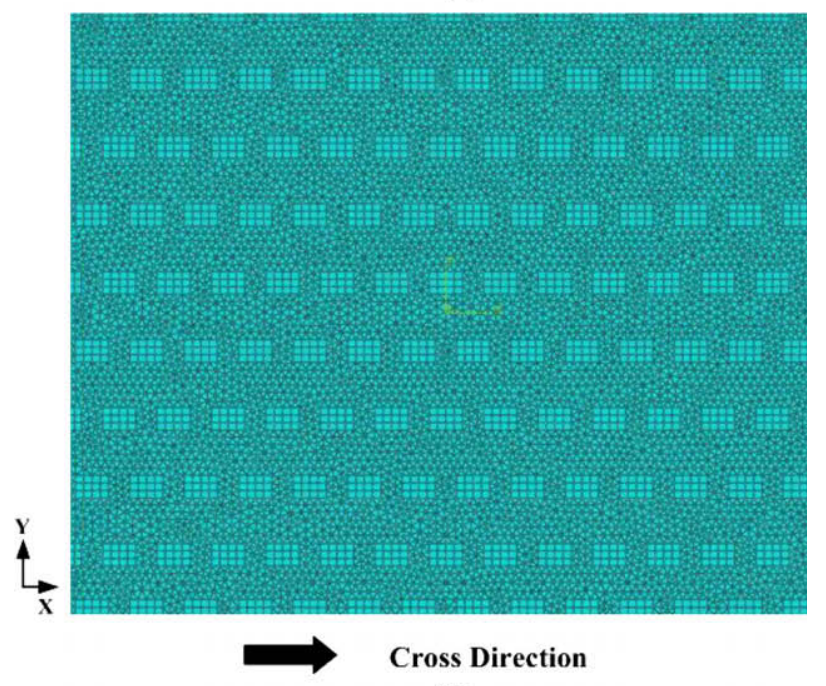

(b)

Fig. 4. Undeformed continuous finite element models: (a) machine direction; (b) cross direction.

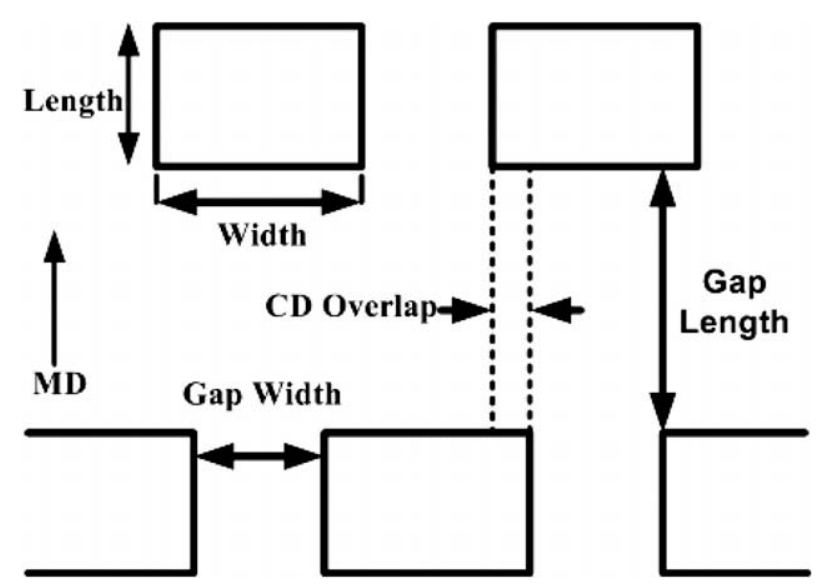

Fig. 5. Layout of bonding points in material.

direction, the model presented a clear striped system, which was formed by lines of bonding points with intermediate stripes of fibrous web. According to the obtained results, the line of bonding 
Table 1

Geometry data for bonding points of nonwoven material.

\begin{tabular}{|c|c|c|c|c|c|}
\hline Bond shape & Width (mm) & Height (mm) & Gap width in CD (mm) & Gap length in MD (mm) & CD overlap (mm) \\
\hline Rectangle & 1.1 & 0.75 & 0.7 & 1.5 & 0.2 \\
\hline
\end{tabular}
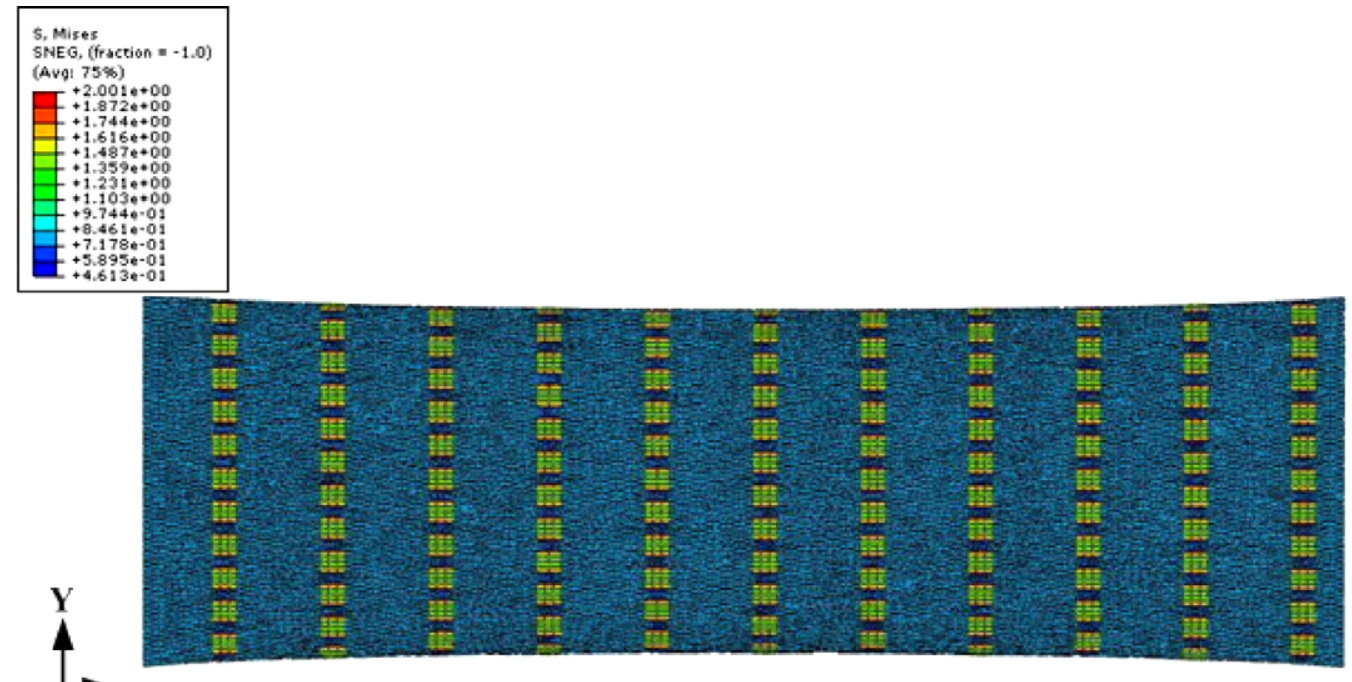

$\mathbf{X}$

(a)

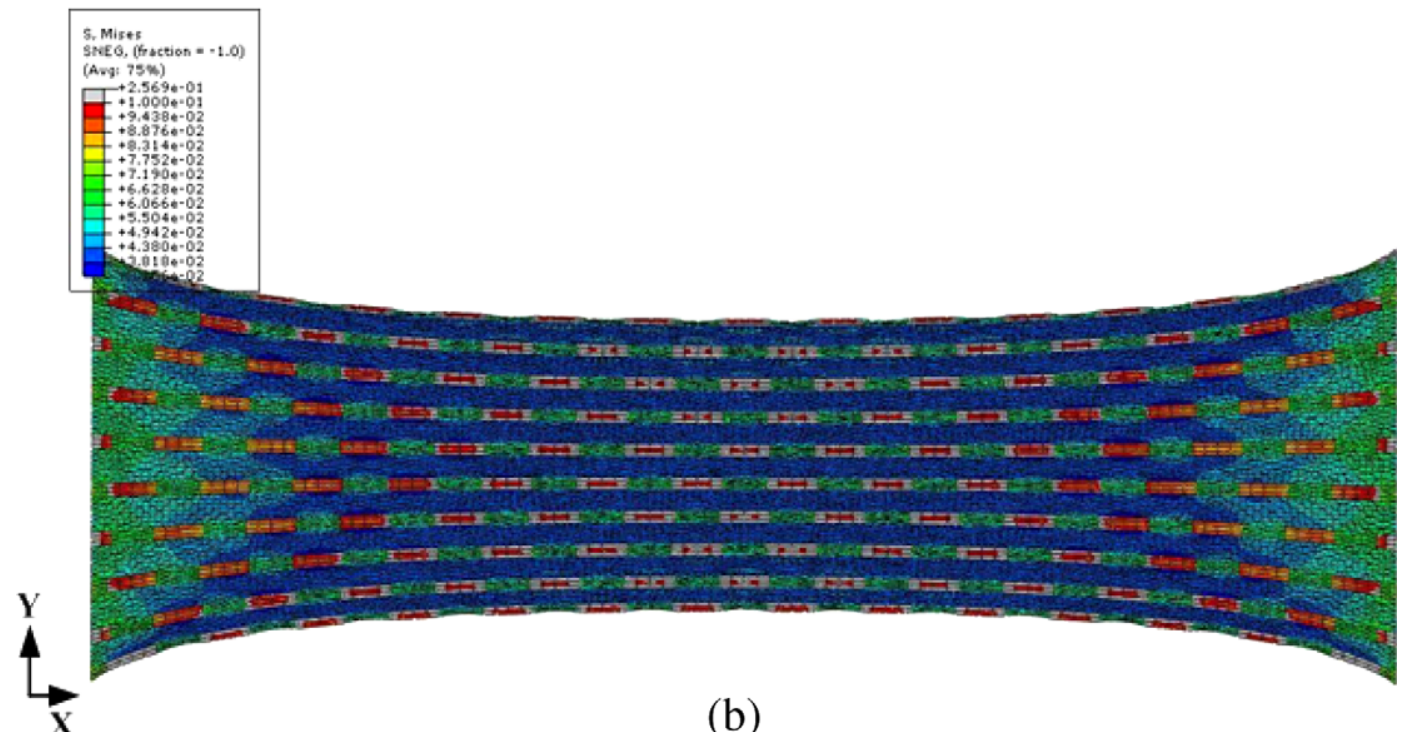

Fig. 6. Deformed continuous FEA models: (a) machine direction; (b) cross direction.

points carries a higher stress level due to their higher stiffness. And the area of fibrous network was characterised by a uniform and relative low stress. In cross direction, the stress distribution was more complicated; the stress in the area of fibrous web is not uniform as in machine direction. Due to the pattern of bonding points, four neighbouring points formed a diamond pattern, which acted as a basic load-carrying unit in the material. During the deformation the stress transfers along the boundaries of the diamond patterns. The obtained results match the experimental observation well. Another result of the simulations is the different necking behaviours for two principle directions. In machine direction, necking of the stretched specimen is much smaller than the one in cross direction due to its higher stiffness in lateral direction. These phenomena also match our experimental observations.

The results for stress-strain relations obtained in simulations with the continuous FE model, which describes the anisotropic material properties of the nonwoven material, are presented in Fig. 7. A comparison between the results of the simulation and experiment in machine direction shows a slower stress increase at initial stages of stretching than in experiments; the experimental and calculated curves have an intersection at approx. $150 \%$ strain. It means that the model predicts the maximum level of load observed in experiments, with materials failing at such a level of strain. The difference for the initial 


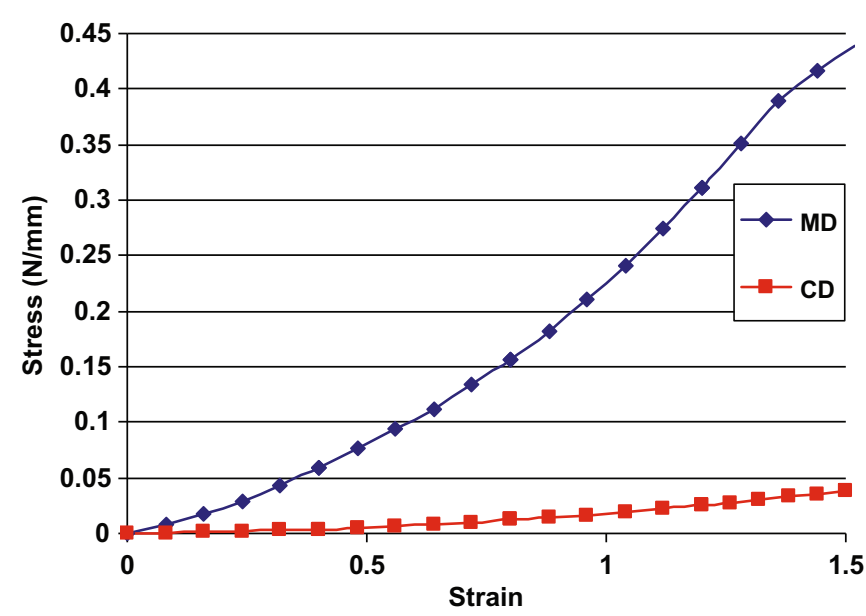

Fig. 7. Calculated stress-strain relationships for MD and CD (continuous FE model).

stages of deformation was due to the underestimation of the real stress by the continuous FEA model since it could not account for deformational mechanisms acting in thermally bonded nonwovens at the micro-scale. Hence, the use of shell elements resulted in the overestimation of the stiffness in the cross sectional area.

The results of numerical simulations in cross direction match the experimental results well; similar stress-strain curves were obtained. This is linked to the fact that for CD the lateral contraction is bigger than for MD due to the arrangement of the bonding points, and the volume change of the specimen is dominated by the lateral compression. Therefore, in cross direction, the effect of void areas does not play an important role here as for the machine direction.

\section{Discontinuous model}

According to the results of our experiments and the initial continuous model, the mechanical properties of the nonwoven material are determined by both the material properties of single fibres and microstructure of the material. To simulate the effect of microstructure of the nonwoven, a discontinuous model was developed to reflect its features.

Geometric information for the fibrous web is the fibre diameter, fibre orientation distribution and curve feature of fibres. According to our experimental results, the fibre orientation distribution is one of the most important factors, which determines the material's anisotropy and discontinuity. And the fibrous network forms the matrix in nonwoven that contains bonding points. Therefore, the present model assumes the fibres are straight and arranged according to orientation distributions. The orientation distribution of the studied fabric was measured at North Carolina State University [11-13]. To generate the random fibrous network, a program was developed using Python language to describe the random fibrous assembly according to their orientation distribution. In this algorithm, to generate one fibre, a point $\mathrm{O}$ (Fig. 8 ) is located as the centre point of a sheet area first, then angle $\alpha$ is defined according to the orientation distribution of the fibre. From the point $\mathrm{O}$, a segment with a slope $\alpha$ and a random length $d$ is generated using random functions. Finally, a line, perpendicular to this segment and intersecting it at the end point, opposite to $O$, is generated throughout the sheet area. By repeating this method, a fibrous network with continuous fibres was generated according to the experimentally measured orientation distribution of fibres. But at this stage of the study, it is not practical to introduce the real number

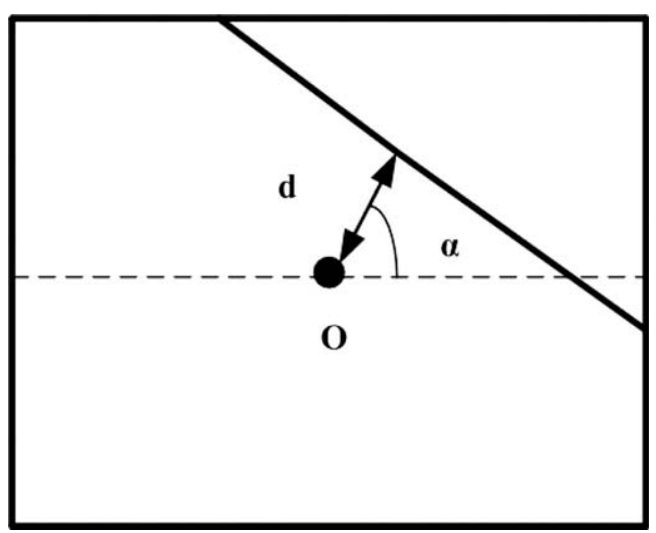

Fig. 8. Scheme for algorithm used to model random distribution of fibres.

of fibres into the model, which could be very large. Therefore, each line in the model represented not a single fibre but a number of fibres having close directions.

Then the coordinates of the end points of the line were calculated and transformed into the format, which could be read by ABAQUS software. After editing the input file of ABAQUS, the geometry of the discontinuous model was generated as shown in Fig. 9a. The dimension of the fibrous web is $25 \mathrm{~mm} \times 20 \mathrm{~mm}$. To investigate the geometric nonlinearity and the effect of bonding points on the anisotropy of the material, 200 representative lines with random lengths were used in present discontinuous model assembled according to the uniform orientation distribution. All the fibres were modelled straight neglecting the effect of their curvature in order to reduce the computational cost and the risk of dissociation.

To introduce the geometric information of bonding points into FE simulations, the geometric model of the fibrous network should be divided into areas to leave the space for the bonding points because overlapping elements cause problems during meshing. The coordinates of the bonding points are used to divide the continuous fibres within the geometry model of the fibrous network; the resulting geometry model for $\mathrm{MD}$ and $\mathrm{CD}$ are shown in Fig. 9b and c, respectively.

Then the geometry of bonding points was used as input into the ABAQUS software according to the measurement data obtained for the real material. And each bonding point was divided into two areas - the central area and the external area $(0.5 \mathrm{~mm}$ wide) along the perimeter of the points - to leave the possibility of combining elements of the fibres and bonding points.

The meshing process of the discontinuous model was carried out in two steps. First, the fibrous network was meshed using truss elements (T2D2) in order to avoid the ABAQUS software generating nodes automatically in the overlapping points of fibres to stop sliding of fibres. The nodes of elements were introduced into the ABAQUS input file directly. Then, the bonding points were meshed using shell elements in ABAQUS (Fig. 10). The external areas of the bonding points were meshed first with spacing between nodes $1 \mathrm{~mm}$. At the next stage, additional nodes along the boundaries of bonding points were defined to coincide with the nodes of truss elements, connected to the boundaries. So there were common nodes shared by both the shell elements and the truss elements, which gave the possibility to transfer load and displacements from fibres to bonding points. The internal areas of bonding points then were meshed according to the location of the shared nodes with the external mesh.

To the authors' knowledge, there are only two discontinuous finite element models in literature used to simulate nonwoven 


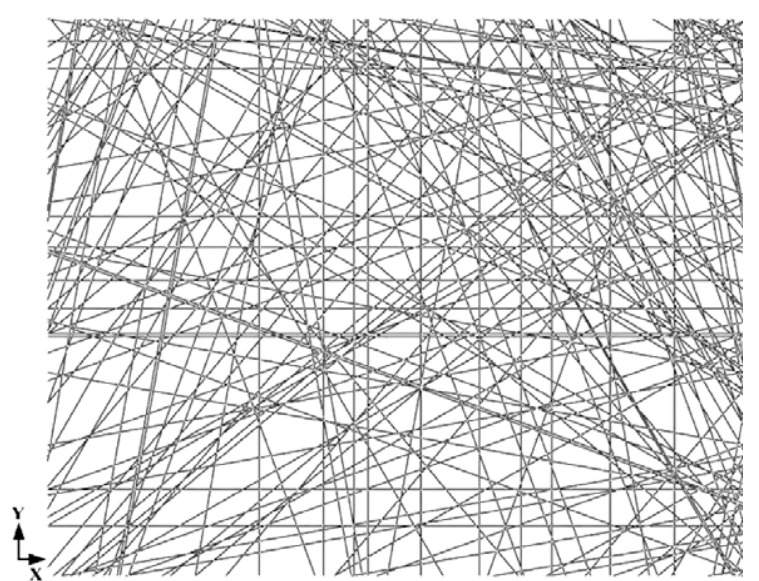

(a)

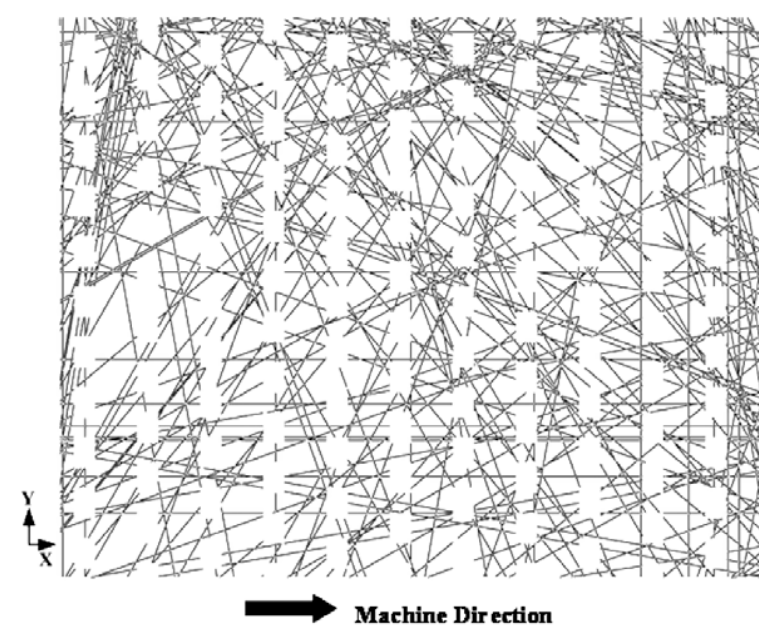

(b)

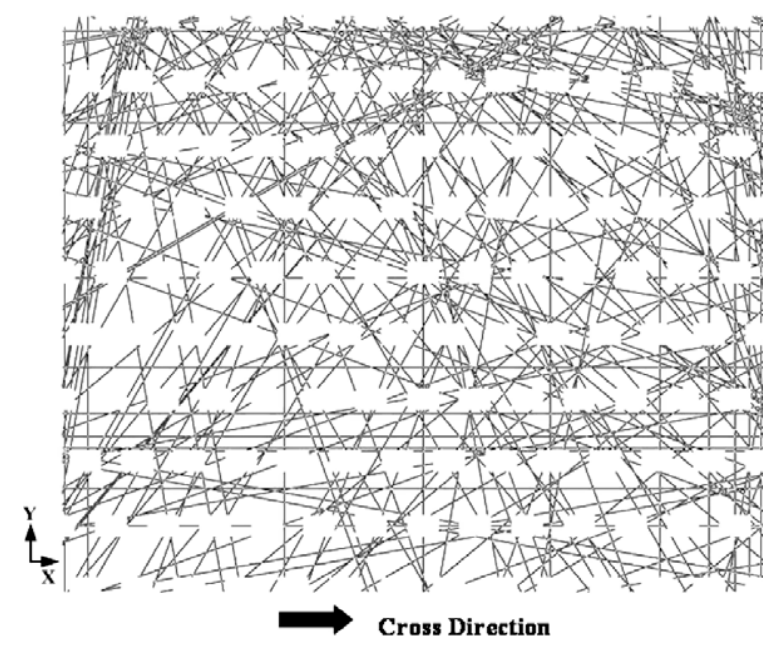

(c)

Fig. 9. Fibrous network of discontinuous model (a) and geometry models of divided fibrous network: machine direction (b) and cross direction (c).

materials. Comparing the mesh details of our model with those in the Mueller's model [19], it is easy to find out the fibres in present model were generated randomly and not periodically. Besides, finer meshes were generated in our model for the bonding points - as compared with 8 elements per point in [19] - so that to connect more fibres to them. Therefore, our model has the capabil-

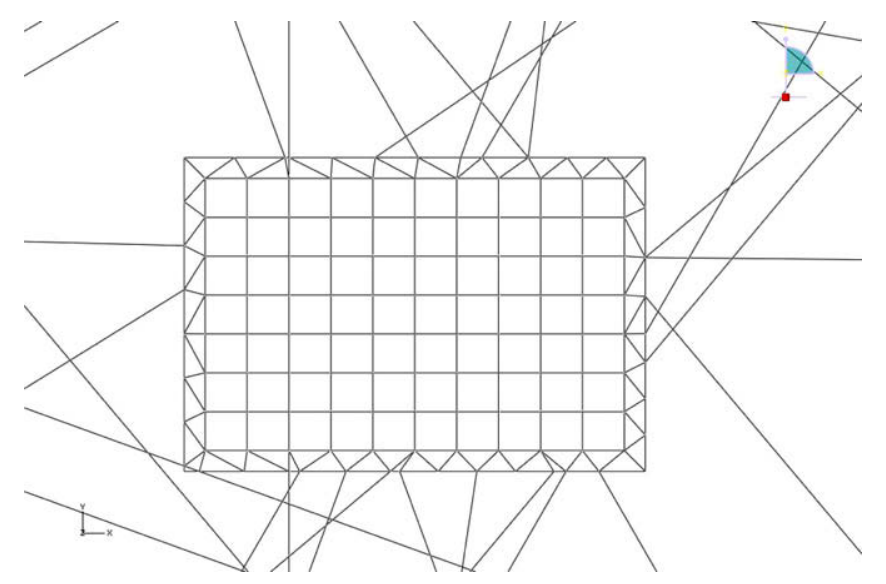

Fig. 10. Meshed details for discontinuous model.

ity to simulate the mechanical properties due to the non-uniformity of the material as well as the mechanical behaviour of bonding points.

The same boundary conditions, used in our continuous FE model, were employed in the discontinuous one.

Results of FE simulations based on the discontinuous model are presented in Fig. 11 for both machine and cross directions. In machine direction, the model can also simulate the stripped system and the deformation effect due to necking during the longitudinal extension. In the initial period of the extension the stress transfers from one bonding line to another. When the strain achieves a relatively high level, the space between neighbouring bonding points will vanish and they start to rotate, distorting the line of bonding points. This behaviour cannot be achieved in the continuous model. Hence, FE simulations better reproduce deformation processes in real nonwovens. And the discontinue model can also describe the non-uniform stress distribution due to the uneven density in the fabric as well as model the areas of high stress concentration in the stretched material. As was observed in experiments, failure of nonwoven materials starts at very early stages of their deformation; some fibres break even when the overall deformation of the material is small due to variations in their lengths and orientations. Therefore, with the discontinuous model, it is possible to find out the network areas and bond points with potentially high risk of damage, and to study the rupture mechanism.

In cross direction, at the initial stage of extension, the bonding lines are the main load-carrying component. At relatively large strain, four neighbouring bonding points forms a diamond pattern as a basic load carrier, which is different from the deformation mechanism in the machine direction. This mechanism could explain the observed material's nonlinear behaviour in the initial period of the extension (Fig. 3).

To investigate deformational features of the generated fibrous network and the effect of bonding points, provisional linear elastic fibre properties were employed at this stage of research together with the same angular distribution of fibres for MD and $C D$. The results of the simulation based on the discontinuous model with these assumptions are shown in Fig. 12; nonlinear stress-strain curves were obtained for both directions. The results demonstrate that the random microstructure causes the nonlinear response of the material, even when constituting fibres have linear properties. Another finding from the simulations is closeness of results obtained for both machine direction and cross direction since MD and $C D$ models in those simulations had the same fibre orientation distribution but various patterns of bonding points. It is obvious that the studied pattern of bonding points does not significantly affect the anisotropy of the material. 


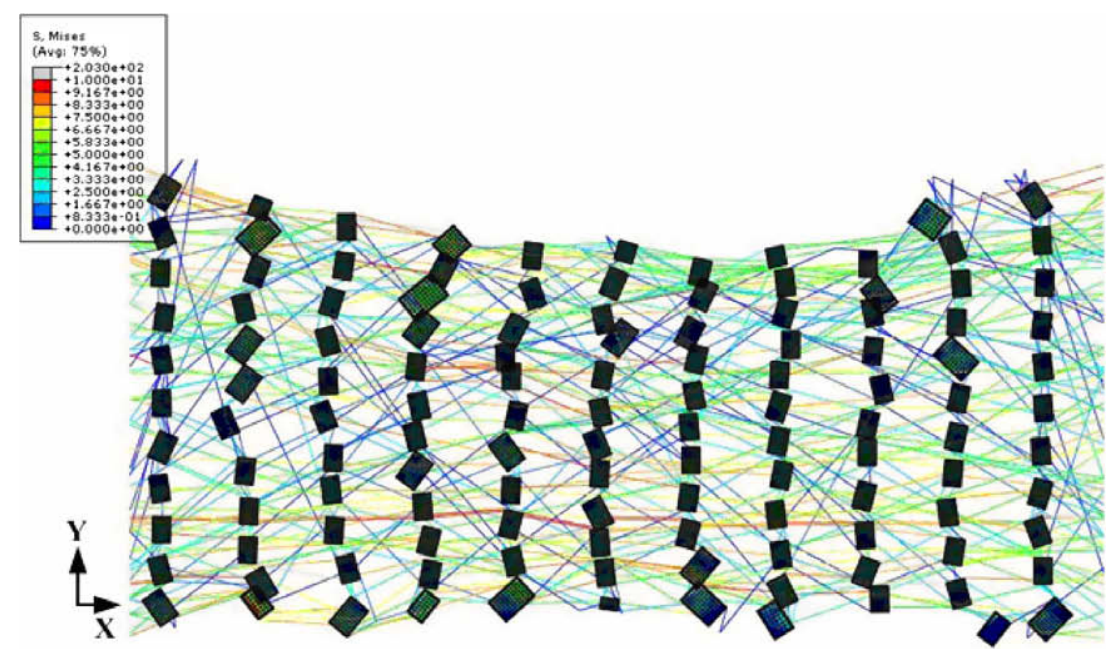

(a)

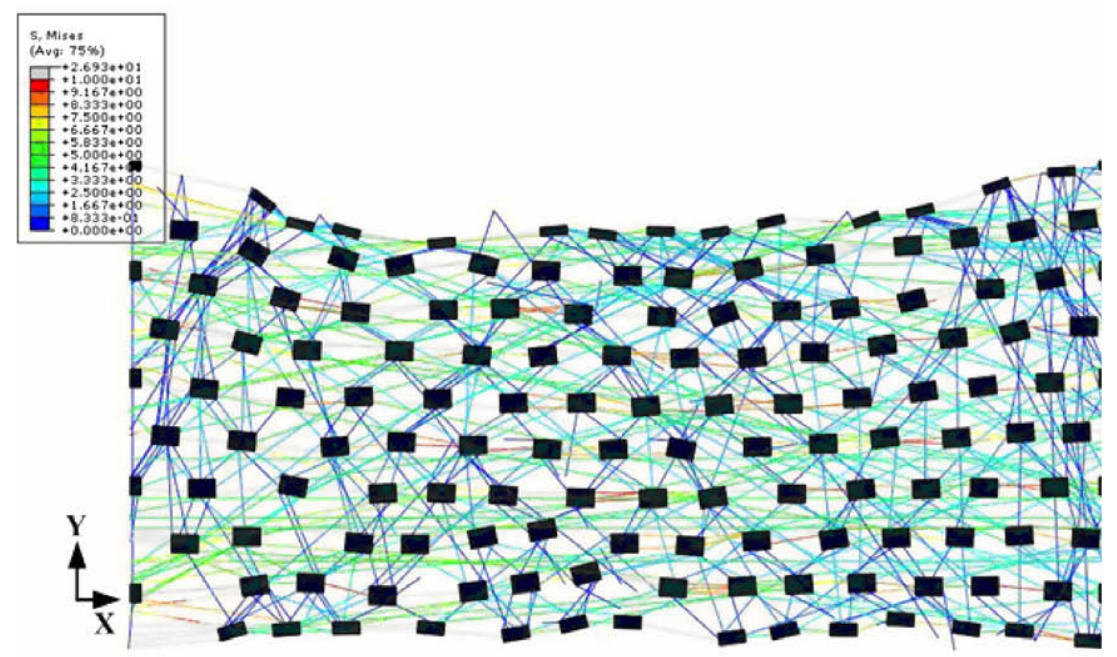

(b)

Fig. 11. Deformed discontinuous models: (a) machine direction; (b) cross direction.

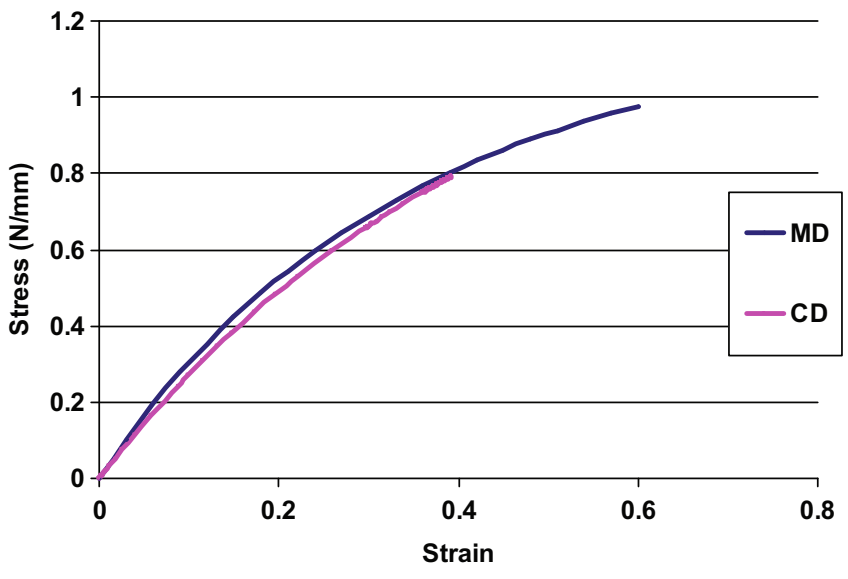

Fig. 12. Nonlinear behaviour of random fibrous webs.

\section{Conclusions}

Two different finite element approaches to modelling of the thermally bonded nonwovens were implemented. The first approach employs continuous models to describe the material based on its macrostructure. The discontinuous models of the second approach incorporate features of the material's microstructure into FE simulations. The obtained results follow:

1. The continuous model presents the material behaviours of nonwovens at macro-scale in both machine direction and cross direction. Although due to its continuous nature the model could not include the discontinuous and non-uniform microstructure of the material, the model still could help to analyse the effects of bonding features (density, shape, size, spacing) on the overall properties of the fabric and deformation mechanisms. And the results of simulations with the model proved that the effect of microstructure can not be ignored for such materials. An advanced model should be developed and account for the effects of material's microstructure.

2. A discontinuous FE model was developed based on the results of the initial continuous one. Besides the material's features at macro-scale, the discontinuous model also directly introduces material's microstructure (density, discontinuity, non-uniformity, orientation distribution and properties of fibres) into simulations by generating the random fibrous network. In this paper, the nonlinearity of the material's response to loading was analysed using the discontinuous model. The random fibrous structure results in the highly nonlinear behaviour of 
the studied material even when the fibres have linear properties due to their relative movements in the network. And the effect of bonding points on the anisotropy of the material has been estimated. Although the pattern of bonding points of the material is direction-dependent, it does not significantly contribute to the anisotropy of the overall material. The discontinuous model provides a strong tool to study the complicated material properties of the nonwoven material. Based on the model it is possible to analyse these features separately that would be impossible in real-life experiments, and to obtain a deeper understanding of material's properties of nonwovens.

\section{Acknowledgment}

We greatly acknowledge the support by the Nonwovens Cooperative Research Center of North Carolina State University, Raleigh, USA.

\section{References}

[1] A. Rawal, Journal of Industrial Textiles 36 (2006) 133-149.

[2] R. Krčma, Nonwoven Textiles, first ed., SNTL, Manchester, 1962.

[3] T. Komori, M. Itoh, Textile Research Journal 61 (10) (1991) 588-594.

[4] M.A. Narter, S.K. Batra, D.R. Buchanan, Proceedings of the Royal Society 455 (1999) 3543-3563.

[5] S. Backer, D.R. Petterson, Textile Research Journal 30 (1960) 704-711.
6] H.S. Kim, Fiber and Polymers 5 (2004) 139-144

[7] S. Bais-Singh, B.C. Goswami, Textile Research Journal 68 (1998) 219-227.

[8] H.S. Kim, B. Pourdeyhimi, Journal of Textile and Appeal, Technology and Management 1 (2000) 1-6.

[9] H.S. Kim, B. Pourdeyhimi, Journal of Textile and Appeal, Technology and Management 1 (2001) 1-7.

[10] H.S. Kim, B. Pourdeyhimi, International Nonwovens Journal 10 (2001) 32-37.

[11] B. Pourdeyhimi, R. Ramanathan, R. Dent, Textile Research Journal 66 (11) (1996) 713-722.

[12] B. Pourdeyhimi, R. Ramanathan, R. Dent, Textile Research Journal 66 (12) (1996) 747-753.

[13] B. Pourdeyhimi, R. Dent, H. Davis, Textile Research Journal 67 (2) (1997) 143151.

[14] H.S. Kim, B. Pourdeyhimi, A. Deshpande, A. Abhiraman, P. Desai, Textile Research Journal 71 (2) (2001) 157-164.

[15] H.S. Kim, B. Pourdeyhimi, P. Desai, A. Abhiraman, Textile Research Journal 71 (11) (2001) 965-976.

[16] H.S. Kim, B. Pourdeyhimi, A. Abhiraman, P. Desai, Textile Research Journal 72 (7) (2002) 645-653.

[17] C.A. Bronkhorst, International Journal of Solids and Structures 40 (2003) 54415454.

[18] M.K. Ramasubramanian, Y. Wang, International Journal of Solids and Structures 44 (2007) 7615-7632.

[19] D.H. Mueller, M. Kochmann, International Nonwovens Journal 13 (2004) 5662.

[20] S. Limem, S.B. Warner, Textile Research Journal 75 (2005) 63-72.

[21] G.C. Engelmayr Jr., M.S. Sacks, Journal of Biomechanical Engineering 128 (2006) 610-622.

[22] X. Hou, M. Acar, V.V. Silberschmidt, Journal of Engineered Fibers and Fabrics 4 (2009) 26-33.

[23] ABAQUS 6.7., User’s Manual, Simulia, 2008. 